\title{
Descent Monomials, $P$-Partitions and Dense Garsia-Haiman Modules
}

EDWARD E. ALLEN

allene@wfu.edu

Department of Mathematics, Wake Forest University, Winston-Salem, NC 27109, USA

Received October 31, 2002; Revised September 19, 2003; Accepted September 29, 2003

\begin{abstract}
A two-variable analogue of the descents monomials is defined and is shown to form a basis for the dense Garsia-Haiman modules. A two-variable generalization of a decomposition of a $P$-partition is shown to give the algorithm for the expansion into this descent basis. Some examples of dense Garsia-Haiman modules include the coinvariant rings associated with certain complex reflection groups.
\end{abstract}

Keywords: descent monomials, $P$-partitions, Garsia-Haiman modules, convariant rings

\section{Introduction}

Let $\mathcal{A}$ denote the infinite collection

$$
\mathcal{A}=\{\ldots,(0,3),(0,2),(0,1),(0,0),(1,0),(2,0),(3,0), \ldots\}
$$

For $\left(a_{1}, b_{1}\right),\left(a_{2}, b_{2}\right), \in \mathcal{A}$, we define $\left(a_{1}, b_{1}\right)<_{\mathcal{A}}\left(a_{2}, b_{2}\right)$ whenever $a_{1}-b_{1}<a_{2}-b_{2}$. Let

$$
S=\left\{\left(a_{1}, b_{1}\right),\left(a_{2}, b_{2}\right), \ldots,\left(a_{n}, b_{n}\right)\right\} \subset \mathcal{A}
$$

denote a finite subset of $\mathcal{A}$ listed so that $\left(a_{i}, b_{i}\right)<_{\mathcal{A}}\left(a_{i+1}, b_{i+1}\right)$. Furthermore, let $M_{S}$ denote the $n \times n$ matrix

$$
M_{S}=\left(x_{i}^{a_{k}} y_{i}^{b_{k}}\right)_{1 \leq i, k \leq n}
$$

and let $\Delta_{S}(X, Y)$ denote the determinant of $M_{S}$. Set $\mathbb{C}[X, Y]$ to be the polynomial ring over the complex field $\mathbb{C}$ with variables in $X=\left\{x_{1}, x_{2}, \ldots, x_{n}\right\}$, and $Y=\left\{y_{1}, y_{2}, \ldots, y_{n}\right\}$. With $P(X, Y) \in \mathbb{C}[X, Y]$, we will set

$$
P\left(\partial_{X}, \partial_{Y}\right)=P\left(\partial_{x_{1}}, \partial_{x_{2}}, \ldots, \partial_{x_{n}}, \partial_{y_{1}}, \partial_{y_{2}}, \ldots, \partial_{y_{n}}\right),
$$

where $\partial_{x_{i}}$ denotes the partial differential operator with respect to $x_{i}$. With

$$
\mathcal{I}_{S}(X, Y)=\left\{P(X, Y) \in \mathbb{C}[X, Y]: P\left(\partial_{X}, \partial_{Y}\right) \Delta_{S}(X, Y)=0\right\},
$$


set $\mathbb{C}_{S}[X, Y]$ to be the polynomial quotient ring

$$
\mathbb{C}_{S}[X, Y]=\mathbb{C}[X, Y] / \mathcal{I}_{S}(X, Y) .
$$

These rings $\mathbb{C}_{S}[X, Y]$ are called Garsia-Haiman modules. A. Garsia and M. Haiman introduced modules of a similar nature to study the Kostka $q, t$ coefficients (see[5]). The ideas, however, can be traced back to Macaulay.

Associated with a subset

$$
S=\left\{\left(0, b_{1}\right),\left(0, b_{2}\right), \ldots,\left(0, b_{j-1}\right),(0,0),\left(a_{j+1}, 0\right), \ldots,\left(a_{n}, 0\right)\right\}
$$

of $\mathcal{A}$, listed in increasing order with respect to $<_{\mathcal{A}}$, are two sequences $\psi_{S}$ and $\Xi_{\psi_{S}}$ defined as follows.

First, for $1 \leq j \leq n-1$, set $\psi_{S}$ to be

$$
\psi_{S}=\left[\left[1, \beta_{2}, \ldots, \beta_{j}\right],\left[1, \alpha_{2}, \ldots, \alpha_{n+1-j}\right]\right],
$$

with $\beta_{1}=\alpha_{1}=1$ and for $2 \leq k \leq j$,

$$
\beta_{k}=b_{j+1-k}-b_{j+2-k} .
$$

and for $2 \leq h \leq n+1-j$,

$$
\alpha_{h}=a_{j+h-1}-a_{j+h-2}
$$

where $b_{j}=0$ and $a_{j}=0$. Collections

$$
\psi_{S}=\left[\left[\beta_{1}, \beta_{2}, \ldots, \beta_{n}\right], \emptyset\right]
$$

or

$$
\psi_{S}=\left[\emptyset,\left[\alpha_{1}, \alpha_{2}, \ldots, \alpha_{n}\right]\right]
$$

will be dealt with in Section 7 .

Second, set

$$
\Xi_{\psi_{S}}=\left\{\left[\rho_{1}, \ldots, \rho_{j-1}, 0, \rho_{j+1}, \ldots, \rho_{n}\right]\right\}_{\rho}
$$

where

$$
0 \leq \rho_{k}<b_{k}-b_{k+1}=\beta_{j+1-k},
$$


for $1 \leq k \leq j-1$, and

$$
0 \leq \rho_{h}<a_{h}-a_{h-1}=\alpha_{h-j+1},
$$

for $j+1 \leq h \leq n$.

For $\sigma \in S_{n}$ (the symmetric group), $\psi=\left[\left[1, \beta_{2}, \ldots, \beta_{j}\right],\left[1, \alpha_{2}, \ldots, \alpha_{n+1-j}\right]\right]$ and $\rho=\left[\rho_{1}, \rho_{2}, \ldots, \rho_{n}\right] \in \boldsymbol{\Xi}_{\psi_{s}}$, define

$$
\begin{aligned}
d_{\sigma, \rho}^{j}(X, Y)= & \left(\prod_{k=1}^{j-1} y_{\sigma(1)}^{\rho_{k}} \cdots y_{\sigma(k)}^{\rho_{k}}\right)\left(\prod_{\substack{1 \leq i \leq j-1 \\
\sigma(i)>\sigma(i+1)}} y_{\sigma(1)} \cdots y_{\sigma(i)}\right) \\
& \times\left(\prod_{h=j+1}^{n} x_{\sigma(h)}^{\rho h} x_{\sigma(h+1)}^{\rho_{h}} \cdots x_{\sigma(n)}^{\rho_{h}}\right)\left(\prod_{\substack{j+1 \leq i \leq n \\
\sigma(i-1)>\sigma(i)}} x_{\sigma(i)} \cdots x_{\sigma(n)}\right) .
\end{aligned}
$$

For example, if $\sigma=(5,8,4,2,3,6,1,7), \rho=[4,0,3,1,0,1,0,7]$ and $j=5$ then

$$
\begin{aligned}
& d_{\sigma, \rho}^{5}(X, Y) \\
& \quad=\left(y_{5}^{4}\right)\left(y_{5}^{0} y_{8}^{0}\right)\left(y_{5}^{3} y_{8}^{3} y_{4}^{3}\right)\left(y_{5}^{1} y_{8}^{1} y_{4}^{1} y_{2}^{1}\right)\left(y_{5} y_{8}\right)\left(y_{5} y_{8} y_{4}\right)\left(x_{6} x_{1} x_{7}\right)\left(x_{7}^{7}\right)\left(x_{1} x_{7}\right) \\
& \quad=x_{1}^{3} y_{2} y_{4}^{5} y_{5}^{10} x_{6} x_{7}^{9} y_{8}^{6} .
\end{aligned}
$$

Note that the terms

$$
\prod_{\substack{1 \leq i \leq j-1 \\ \sigma(i)>\sigma(i+1)}} y_{\sigma(1)} \cdots y_{\sigma(i)}
$$

and

$$
\prod_{\substack{j+1 \leq i \leq n \\ \sigma(i-1)>\sigma(i)}} x_{\sigma(i)} \cdots x_{\sigma(n)}
$$

are partial terms of descent monomials and that

$$
\left(\prod_{k=1}^{j-1} y_{\sigma(1)}^{\rho_{k}} \cdots y_{\sigma(k)}^{\rho_{k}}\right)\left(\prod_{\substack{1 \leq i \leq j-1 \\ \sigma(i)>\sigma(i+1)}} y_{\sigma(1)} \cdots y_{\sigma(i)}\right)
$$

and

$$
\left(\prod_{h=j+1}^{n} x_{\sigma(h)}^{\rho_{h}} x_{\sigma(h+1)}^{\rho_{h}} \cdots x_{\sigma(n)}^{\rho_{h}}\right)\left(\prod_{\substack{j+1 \leq i \leq n \\ \sigma i-1>\sigma(i)}} x_{\sigma(i)} \cdots x_{\sigma(n)}\right)
$$


are correspondingly partial terms in the $P$-partition decomposition given in [10] (see page 213).

The purpose of this paper is to prove the following theorem. Note that the definition of a dense sequence will be given in Section 2. Also, recall that $j$ is implicitly defined in $S$.

Theorem 1.1 Let $S$ be a dense sequence. The collection

$$
\mathcal{D}_{S}=\left\{d_{\sigma, \rho}^{j}(X, Y): \sigma \in S_{n}, \rho \in \Xi_{\psi_{S}}\right\}
$$

is a basis for $\mathbb{C}_{S}[X, Y]$ with coefficients from $\mathbb{C}$.

Note that Theorem 1.1 is a generalization of a well-studied theorem. If $\psi=[[1, \ldots$, $1,1],[1]]$ then $j=n$ and

$$
\Xi_{\psi_{S}}=\{[0,0, \ldots, 0]\}
$$

The only possible choice for $\rho$ is

$$
\rho=[0,0, \ldots, 0]
$$

In this case, $d_{\sigma, \rho}^{j}(X, Y)$ reduces to the normal descent monomial

$$
d_{\sigma, \rho}^{j}(X, Y)=\prod_{\sigma_{i}>\sigma_{i+1}} y_{\sigma_{1}} \cdots y_{\sigma_{i}}
$$

and $\mathcal{D}_{S}$ becomes the collection of descent monomials in the variables $Y$. Furthermore, $\Delta_{S}(X, Y)$ becomes the Vandermonde determinant in $Y$ and $\mathcal{I}_{S}(X, Y)$ becomes the ideal generated by the elementary symmetric functions in the variables $Y$ and $\left\{x_{1}, x_{2}, \ldots, x_{n}\right\}$. Essentially, $\mathbb{C}_{S}[X, Y]$ is the coinvariant ring (i. e., $\mathbb{C}\left[y_{1}, y_{2}, \ldots, y_{n}\right] / I$ where $I$ is the ideal generated by the elementary symmetric functions in the variables $Y$ ) associated with the symmetric group $S_{n}$. In [1,4] and [8], various proofs have been given that show that the descent monomials are a basis for $C\left[y_{1}, y_{2}, \ldots, y_{n}\right] / I$.

Additionally, with $1 \leq h \leq g$ and

$$
\psi_{S}=[[h, g, \ldots, g, g], \emptyset],
$$

the ideal $\mathcal{I}_{S}(X, Y)$ is generated by the following symmetric polynomials

$$
e_{k}=\sum_{i_{1}<i_{2}<\cdots<i_{k}} y_{i_{1}}^{g} y_{i_{2}}^{g} \cdots y_{i_{k}}^{g}
$$

for $1 \leq k \leq n-1$ and

$$
e_{n}=y_{1}^{h} y_{2}^{h} \cdots y_{n}^{h} .
$$


The rings $\mathbb{C}_{S}[X, Y]$ are the coinvariant rings associated with certain complex reflection groups-(i.e., $C_{g} 2 S_{n}$ and some particular subgroups of $C_{g} 2 S_{n}$, where $C_{g}$ denotes the cyclic group of order $g$ - see, for example, [2] for two sets of variables $X$ and $Y$ or [7, 10], or [11] for the one variable $X$ case. For example, when the sequence $\psi_{S}=[[2,2,2, \ldots, 2], \emptyset]$ the module $\mathbb{C}_{S}[X, Y]$ corresponds to the coinvariant ring associated to the hyperoctahedral group. These rings in a single variable have been studied by various individuals. For example, in [11] the Hilbert Series of $\mathbb{C}_{S}[X, Y]$ was calculated (with $\psi_{S}$ given in Eq. (1.9)). Morita and Yamada studied the one variable case using bideterminants in [7]. In [2], a bipermanent basis was constructed over variables $X$ and $Y$. As we will see, $\psi_{S}=[[h, g, \ldots, g, g], \emptyset]$ is an example of certain sequences that we will call dense (see Section 2). Thus, the results in this paper apply not only to the coinvariant rings associated to these certain complex reflection groups but also to a much larger classification of rings. Particularly, this paper deals with a certain two-variable generalization of these rings and an extension of the theory of descent monomials and $P$-Partitions to a broader class of rings, specifically the class of dense Garsia-Haiman modules. A generalization of a $P$-Partition decomposition gives us the needed combinatorics to prove the necessary theorems.

In Section 2, dense Garsia-Haiman modules will be defined. In Section 3 we will review some results about cocharge tableaux and the Hilbert series of $\mathbb{C}_{S}[X, Y]$ and prove that a summation of a statistic over the collection of descent monomials gives the Hilbert series for $\mathbb{C}_{S}[X, Y]$. In Section 4, we will identify some particular polynomials in the ideal $\mathcal{I}_{S}(X, Y)$. In Section 5, we will describe a decomposition that is a generalization of the $P$-partition decomposition. In Section 6, we will prove that the descent monomials given in Eq. (1.8) are a basis for $\mathbb{C}_{S}[X, Y]$. Specifically, we will give an algorithm for expressing a monomial $P(X, Y)$ as a linear combination of elements of $\mathcal{D}_{S}$. In Section 7, we will look at analogous results for dense one-variable Garsia-Haiman modules.

\section{Dense sequences}

Recall that

$$
\psi_{S}=\left[\left[1, \beta_{2}, \ldots, \beta_{j}\right],\left[1, \alpha_{2}, \ldots, \alpha_{n+1-j}\right]\right] .
$$

We will consider $\alpha_{1}=\beta_{1}=1$. For $1 \leq k \leq j$, set

$$
f_{k}=-1+\sum_{i=1}^{k} \beta_{i}
$$

and for $1 \leq h \leq n+1-j$, set

$$
g_{h}=-1+\sum_{i=1}^{h} \alpha_{i} .
$$

Note that $f_{k}=b_{j-k+1}$ and $g_{h}=a_{j+h-1}$ where $f_{k}, g_{h}, b_{j-k+1}$ and $a_{j+h-1}$ are as defined in Eqs. (1.5), (2.2) and (2.3). We will say that $\psi_{S}$ is dense if and only if both of the following two conditions hold. 
1. For all $j$ such that $1 \leq k \leq j$ and for all sequences $c_{k}, \ldots, c_{j}$ of nonnegative integers not all zero, either

$$
f_{k}-\sum_{i=k}^{j} c_{i} \beta_{i}<0,
$$

or

$$
f_{k}-\sum_{i=k}^{j} c_{i} \beta_{i}=f_{p},
$$

for some $p$.

2. For all $k$ such that $1 \leq k \leq n-j+1$ and for all sequences $d_{k}, \ldots, d_{n-j+1}$ of nonnegative integers not all zero, either

$$
g_{k}-\sum_{i=k}^{n-j+1} d_{i} \alpha_{i}<0,
$$

or

$$
g_{k}-\sum_{i=k}^{n-j+1} d_{i} \alpha_{i}=g_{q},
$$

for some $q$.

The collection of dense sequences is somewhat extensive. For example, any sequence of the form

$$
\psi_{s}=[[1, g, g, \ldots, g],[1, h, h, \ldots, h]]
$$

(with both $g, h \geq 1$ ) is dense. Another possibility is

$$
\psi_{s}=\left[\left[1, \beta_{2}, \ldots, \beta_{j}\right],\left[1, \alpha_{2}, \ldots, \alpha_{n+1-j}\right]\right]
$$

where $\beta_{k} \mid \beta_{k+1}$ for $1 \leq k \leq j-1$ and $\alpha_{h} \mid \alpha_{h+1}$ for $2 \leq h \leq n-j$. A third example occurs when we require $\alpha_{k} \geq \sum_{i=1}^{k-1} \alpha_{i}$ for $1 \leq k \leq n+1-j$ or $\beta_{k} \geq \sum_{i=1}^{k-1} \beta_{i}$ for $1 \leq k \leq j$. Many types of examples exist. Furthermore, note that being dense is a function of the sequences $\left[1, \beta_{2}, \ldots, \beta_{j}\right]$ and $\left[1, \alpha_{2}, \ldots, \alpha_{n+1-j}\right]$ separately. Thus in these previous example, we could have interchanged a $\left[1, \beta_{2}, \ldots, \beta_{j}\right]$ from one example into another and the resulting sequence

$$
\psi_{s}=\left[\left[1, \beta_{2}, \ldots, \beta_{j}\right],\left[1, \alpha_{2}, \ldots, \alpha_{n+1-j}\right]\right]
$$

would have remained dense.

We will say that the Garsia-Haiman module $\mathbb{C}_{S}[X, Y]$ is dense, whenever the subset $S$ is dense. 


\section{Cocharge tableaux and the Hilbert series of $\mathbb{C}_{S}[X, Y]$}

We will use French notation to denote ferrers diagrams and taleaux. Let $\lambda=\left(\lambda_{1}, \lambda_{2}, \ldots\right.$, $\lambda_{h}$ ) be a partition of $n$. Specifically, we have that $\lambda_{1} \geq \lambda_{2} \geq \cdots \geq \lambda_{h}>0$ and $n=$ $\lambda_{1}+\lambda_{2}+\cdots+\lambda_{h}$. A ferrers diagram of shape $\lambda$ has $\lambda_{1}$ cells in its first row, $\lambda_{2}$ cells in its second row and, in general, $\lambda_{k}$ cells in its $k$ th cell as $1 \leq k \leq h$. A tableau of shape $\lambda$ is a ferrers diagram of shape $\lambda$ where the cells contain entries from some ordered alphabet. A standard tableau is a tableau where the entries are taken from the set $\{1,2, \ldots, n\}$, each entry appears exactly once and the entries strictly increase from left to right (west to east) in each row and from bottom to top (south to north) in each column. Let $\operatorname{sh}(T)$ denote the shape of $T$.

Define

$$
\delta(i)= \begin{cases}1 & \text { if } i \text { is southeast of } i+1 \\ 0 & \text { if } i \text { is northwest of } i+1\end{cases}
$$

By southeast, we mean strictly south and weakly east. Similarly, by northwest, we mean strictly west and weakly north.

With

$$
\begin{aligned}
\psi_{S} & =\left[\left[1, \beta_{2}, \ldots, \beta_{j}\right],\left[1, \alpha_{2}, \ldots, \alpha_{n+1-j}\right]\right], \\
\rho & =\left[\rho_{1}, \rho_{2}, \ldots, \rho_{n}\right] \in \Xi_{\psi_{S}}
\end{aligned}
$$

and a standard tableau $T$, we can construct a cocharge tableau $C=C_{\rho}(T)$ with $\operatorname{sh}(C)=$ $\operatorname{sh}(T)$ in the following manner (see [3]).

A. Replace $j$ in $T$ by $(0,0)$ and set $c_{j}=\left(c_{j, 1}, c_{j, 2}\right)=(0,0)$.

B. Assuming that we have replaced $h$ in $T$ by $\left(c_{h, 1}, 0\right)$ (for some $h \geq j$ ), replace $h+1$ in $T$ by $\left(c_{h, 1}+\rho_{h+1}+\delta(h), 0\right)$.

C. Assuming that we have replaced $g$ in $T$ by $\left(0, c_{g, 2}\right)$ (for some $g \leq j$ ), replace $g-1$ in $T$ by $\left(0, c_{g, 2}+\rho_{g-1}+\delta(g-1)\right)$.

Note that $C_{\rho}(T)$ has entries from the alphabet $\mathcal{A}$ and that the entries of $C_{\rho}(T)$ increase strictly from south to north and increase weakly from west to east (with respect to $<_{\mathcal{A}}$ ).

Example Let $T$ be the standard tableau of shape $(5,4,3)$

$$
\begin{array}{rcccc}
6 & 9 & 10 & & \\
T=3 & 5 & 7 & 12 & \\
1 & 2 & 4 & 8 & 11
\end{array}
$$

With

$$
\psi_{S}=[[1,1,2,5,5,10],[1,2,2,2,6,6,12]]
$$


and

$$
\rho=[2,3,3,0,1,0,0,1,0,2,4,7],
$$

we have $n=12, j=6$ and

$$
\begin{aligned}
& (0,0) \quad(2,0) \quad(4,0) \\
& C_{\rho}(T)=(0,6) \quad(0,2) \quad(0,0) \quad(16,0) \quad . \\
& (0,12) \quad(0,10) \quad(0,3) \quad(1,0) \quad(8,0)
\end{aligned}
$$

Let $\left|C_{\rho, 1}(T)\right|$ and $\left|C_{\rho, 2}(T)\right|$ denote the sum of the first coordinate entries and the second coordinate entries, respectively, of $C_{\rho}(T)$, With $C_{\rho}(T)$ given in Eq. (3.1) we have $\left|C_{\rho, 1}(T)\right|=31$ and $\left|C_{\rho, 2}(T)\right|=33$, respectively.

Given a graded module $\mathbf{R}$ in the variables $X$ and $Y$, we will let $\mathbf{R}_{s, r}$ denote the homogeneous subspace of total degree $s$ in the variables $X=\left\{x_{1}, x_{2}, \ldots, x_{n}\right\}$ and of total degree $r$ in $Y=\left\{y_{1}, y_{2}, \ldots, y_{n}\right\}$. The Hilbert Series $\mathcal{H}(\mathbf{R})$ of $\mathbf{R}$ is defined as

$$
\mathcal{H}(\mathbf{R})=\sum_{s, t} \operatorname{dim}\left(\mathbf{R}_{s, r}\right) t^{s} q^{r}
$$

The following theorem is proved in [3].

Theorem 3.1 If $S$ is dense then the Hilbert Series $\mathcal{H}\left(\mathbb{C}_{S}[X, Y]\right)$ is given by

$$
\begin{aligned}
\mathcal{H}\left(\mathbb{C}_{S}[X, Y]\right) & =\sum_{\lambda \vdash n,} \sum_{U, T \in \mathcal{S} \mathcal{T}_{\lambda}} \sum_{\rho \in \Xi_{\psi_{S}}} t^{\left|C_{\rho, 1}(T)\right|} q^{\left|C_{\rho, 2}(T)\right|} \\
& =\sum_{\lambda \vdash n} h_{\lambda} \sum_{T \in \mathcal{S} \mathcal{T}_{\lambda}} \sum_{\rho \in \Xi_{\psi_{S}}} t^{\left|C_{\rho, 1}(T)\right|} q^{\left|C_{\rho, 2}(T)\right|}
\end{aligned}
$$

where $\mathcal{S} \mathcal{T}_{\lambda}$ denotes the collection of standard tableaux of shape $\lambda, h_{\lambda}$ denotes the number of standard tableaux of shape $\lambda$ and $\left|C_{\rho, 1}(T)\right|$ and $\left|C_{\rho, 2}(T)\right|$ denote the sum of the first and second coordinates, respectively, of the entries of $C_{\rho}(T)$.

Let $\left(P_{\sigma}, T_{\sigma}\right)$ denote the pair of standard tableaux that we obtain by the row insertion algorithm corresponding to the permutation $\sigma \in S_{n}$ (see [6] or [9], note, however, that we are using the French notation for describing our tableaux). We denote the relationship between a permutation $\sigma \in S_{n_{R}}$ and a pair of standard tableaux $\left(P_{\sigma}, T_{\sigma}\right)$ induced by the row-insertion algorithm by $\sigma \stackrel{R-S}{\longrightarrow}\left(P_{\sigma}, T_{\sigma_{R}}\right)$.

With $\sigma=\left(\sigma_{1}, \sigma_{2}, \ldots, \sigma_{n}\right) \in S_{n}$ and $\sigma \stackrel{R-S}{\longrightarrow}\left(P_{\sigma}, T_{\sigma}\right)$, note that if $\sigma(i)>\sigma(i+1)$ then in $T_{\sigma}$ the cell containing $i$ is southeast (strictly south and weakly east) of the cell containing $i+1$ (follow the bumping path as described in [6]). Similarly, if $\sigma(i)<\sigma(i+1)$ then in $T_{\sigma}$ the cell containing $i$ is northwest (weakly north and strictly west) of the cell containing 
$i+1$. The entry in $C_{\rho}\left(T_{\sigma}\right)$ that replaced $j$ in $T_{\sigma}$ is $(0,0)$. Not that the exponents of $x_{\sigma(j)}$ and $y_{\sigma(j)}$ in $d_{\sigma, \rho}^{j}(X, Y)$ (see Eq. (1.7) are both precisely 0.

Now assume that $\left(c_{m}, 0\right)$ replaced $m$ (for some $m$ such that $\left.j \leq m \leq n-1\right)$ in $T_{\sigma}$ and that $c_{m}$ is the exponent of $x_{\sigma_{m}}$ and 0 is the exponent of $y_{\sigma}(m)$ in $d_{\sigma, \rho}^{j}(X, Y)$.

If $m$ is southeast of $m+1$ in $T_{\sigma}$ then in $C_{\rho}\left(T_{\sigma}\right),\left(c_{m}+\rho_{m+1}+1,0\right)$ replaced the entry $m+1$ of $T_{\sigma}$. If $m$ is southeast of $m+1$ in $T_{\sigma}$, then $\sigma(m)>\sigma(m+1)$ and the exponent of $x_{\sigma(m+1)}$ in $d_{\sigma, \rho}^{j}(X, Y)$ is $c_{m}+\rho_{m+1}+1$. The exponent of $y_{\sigma(m+1)}$ in $d_{\sigma, \rho}^{j}(X, Y)$ is 0 .

If $m$ is northwest of $m+1$ in $T_{\sigma}$, then $\left(c_{m}+\rho_{m+1}, 0\right)$ replaced the entry $m+1$ of $T_{\sigma}$. Furthermore, $\sigma(m)<\sigma(m+1)$ and the exponent of $x_{\sigma(m+1)}$ is $c_{m}+\rho_{m+1}$ and the exponent of $y_{\sigma(m+1)}$ is 0 in $d_{\sigma, \rho}^{j}(X, Y)$.

Similar situations occur for $m$ when $2 \leq m \leq j$ and in which we consider the entry $c_{m-1}$ in $C_{\rho}\left(T_{\sigma}\right)$ and the exponent of $x_{\sigma(m-1)}$ and $y_{\sigma(m-1)}$ in $d_{\sigma, \rho}^{j}(X, Y)$.

Thus if $(a, b)$ is the entry that replaced $i$ in $T_{\sigma}$ when we constructed $C_{\rho}\left(T_{\sigma}\right)$, then the exponents of $x_{\sigma(i)}$ and $y_{\sigma(i)}$ in $d_{\sigma, \rho}^{j}$ are precisely $a$ and $b$, respectively. Therefore, if $\sigma \stackrel{R-S}{\longrightarrow}\left(P_{\sigma}, T_{\sigma}\right)$ then $\left|C_{\rho, 1}\left(T_{\sigma}\right)\right|=\left|d_{\sigma, \rho, 1}^{j}\right|$ and $\left|C_{\rho, 2}\left(T_{\sigma}\right)\right|=\left|d_{\sigma, \rho, 2}^{j}\right|$, where $\left|d_{\sigma, \rho, 1}^{j}\right|$ and $\left|d_{\sigma, \rho, 2}^{j}\right|$ denotes the sum of the exponents in the variables $X=\left\{x_{1}, x_{2}, \ldots, x_{n}\right\}$ and $Y=\left\{y_{1}, y_{2}, \ldots, y_{n}\right\}$ of $d_{\sigma, \rho}^{j}(X, Y)$, respectively. Thus,

Theorem 3.2 If $S$ is dense then the Hilbert Series $\mathcal{H}\left(\mathbb{C}_{S}[X, Y]\right)$ is given by

$$
\begin{aligned}
\mathcal{H}\left(\mathbb{C}_{S}[X, Y]\right) & =\sum_{\lambda \vdash n} \sum_{U, T \in \mathcal{S} \mathcal{T}_{\lambda}} \sum_{\rho \in Z_{\psi_{S}}} t^{\left|C_{\rho, 1}(T)\right|} q^{\left|C_{\rho, 2}(T)\right|} \\
& =\sum_{d_{\sigma, \rho}^{j} \in \mathcal{D}_{S}} t^{\left|\alpha_{\sigma, \rho, 1}^{j}\right|} q^{\left|d_{\sigma, \rho, 2}^{j}\right|}
\end{aligned}
$$

where $\left|d_{\sigma, \rho, 1}^{j}\right|$ and $\left|d_{\sigma, \rho, 2}^{j}\right|$ denote the sum of the exponents of the variables in $X$ and $Y$ respectively, of $d_{\sigma, \rho}^{j}$.

Thus to prove Theorem 1.1 all we need to do is show that the collection $\mathcal{D}_{S}$ spans $\mathbb{C}_{S}[X, Y]$. To do so, we will need to identify some polynomials in the ideal $\mathcal{I}_{S}(X, Y)$ (recall Eq. (1.3)). This is the object of Section 4.

It should be noted that in [3], it is shown that the Hilbert series $\mathcal{H}\left(\mathbb{C}_{S}[X, Y]\right)$ of $\mathbb{C}_{S}[X, Y]$ is given by

$$
\mathcal{H}\left(\mathbb{C}_{S}[X, Y]\right)=f_{\psi_{s}}(q, t) \mathcal{H}\left(\mathbb{C}_{S_{\psi}}[X, Y]\right)
$$

where

$$
f_{\psi_{S}}(q, t)=\prod_{i=1}^{j}\left(\frac{1-q^{(j+1-i) \beta_{i}}}{1-q^{j+1-i}}\right) \prod_{i=1}^{n+1-j}\left(\frac{1-t^{(n+2-j-i) \alpha_{i}}}{1-t^{n+2-j-i}}\right)
$$

and $\mathcal{H}\left(\mathbb{C}_{S_{\Psi}}[X, Y]\right)$ is the Hilbert series corresponding to shape $\Psi=\left[\left[1^{j}\right],\left[1^{n+1-j}\right]\right]$. 


\section{Some polynomials in $\mathcal{I}_{S}(X, Y)$}

With $\gamma_{i}=\left(\gamma_{i, 1}, \gamma_{i, 2}\right)$ and $\gamma=\left[\gamma_{1}, \gamma_{2}, \ldots, \gamma_{n}\right]$ a sequence of length $n$ with entries from $\mathcal{A}$, we set

$$
m_{\gamma}(X, Y)=\sum_{v \in S_{n}} x_{1}^{\gamma_{v(1), 1}} y_{1}^{\gamma_{v(1), 2}} x_{2}^{\gamma_{v(2), 1}} y_{2}^{\gamma_{v(2), 2}} \cdots x_{n}^{\gamma_{v(n), 1}} y_{n}^{\gamma_{v(n), 2}}
$$

Essentially, we are permuting the exponents in Eq. (4.1) (defining $m_{\gamma}(X, Y)$ in this manner becomes very useful in Eq. (4.6) and (6.2)). Note that

$$
\sigma m_{\gamma}(X, Y)=m_{\gamma}(X, Y)
$$

for all $\sigma \in S_{n}$ where the action of $S_{n}$ on a polynomial $P(X, Y)$ is defined by setting

$$
\sigma P\left(x_{1}, x_{2}, \ldots, x_{n}, y_{1}, y_{2}, \ldots, y_{n}\right)=P\left(x_{\sigma(1)}, x_{\sigma(2)}, \ldots, x_{\sigma(n)}, y_{\sigma(1)}, y_{\sigma(2)}, \ldots, y_{\sigma(n)}\right) .
$$

With

$$
\psi_{S}=\left[\left[1, \beta_{2}, \ldots, \beta_{j}\right],\left[1, \alpha_{2}, \ldots, \alpha_{n+1-j}\right]\right],
$$

set $\Gamma_{\psi_{S}}$ to be the collection of sequences

$$
\begin{aligned}
\Gamma_{\psi_{s}}= & \left\{\left[e_{j}, e_{j-1}, \ldots, e_{2},(0,0), h_{2}, \ldots, h_{n+1-j}\right]\right. \\
& \left.: \varepsilon_{2}+\cdots+\varepsilon_{j}+\theta_{2}+\cdots+\theta_{n+1-j}>0\right\},
\end{aligned}
$$

where each $\varepsilon_{i}$ and each $\theta_{i}$ is a nonnegative integer, and

$$
e_{k}=\left(0, \sum_{i=2}^{k} \varepsilon_{i} \beta_{i}\right)
$$

(for $2 \leq k \leq j$ ) and

$$
h_{g}=\left(\sum_{i=2}^{g} \theta_{i} \alpha_{i}, 0\right)
$$

(for $2 \leq g \leq n+1-j$ ).

Theorem 4.1 If $S$ is dense and if $\gamma \in \Gamma_{\psi_{S}}$ then

$$
m_{\gamma}(X, Y) \in \mathcal{I}_{S}(X, Y) .
$$


Proof: $\quad$ Recall that $\mathcal{I}_{S}(X, Y)$ is defined in Eq. (1.3). With $f_{k}$ and $g_{h}$ defined as in Eqs. (2.2) and (2.3), and

$$
\gamma=\left[e_{j}, e_{j-1}, \ldots, e_{2},(0,0), h_{2}, \ldots, h_{n+1-j}\right]=\left[\gamma_{1}, \gamma_{2}, \ldots, \gamma_{n}\right] \in \Gamma_{\psi_{s}},
$$

it is not difficult to see that

$$
\begin{aligned}
& m_{\gamma}\left(\partial_{X}, \partial_{Y}\right) \Delta_{S}(X, Y) \\
& =\sum_{\nu \in S_{n}} c_{\nu} \sum_{w \in S_{n}} \operatorname{sign}(\omega) \omega\left(x_{1}^{0-\gamma_{v}(1), 1} y_{1}^{f_{j}-\gamma_{v}(1), 2} \cdots x_{j-1}^{0-\gamma_{v}(j-1), 1} y_{j-1}^{f_{1}-\gamma_{v}(j-1), 2} x_{j}^{0-\gamma_{v}(j), 1}\right. \\
& \left.\quad y_{j}^{0-\gamma_{v}(j), 2} x_{j+1}^{g_{1,1}-\gamma_{v}(j+1), 1} y_{j+1}^{0-\gamma_{v(j+1), 2}} \cdots x_{n}^{g_{n-j+1,1}-\gamma_{v(n), 1}} y_{n}^{0-\gamma_{v(n), 2}}\right),
\end{aligned}
$$

where $c_{\nu}=0$ if any of the exponents are negative.

Suppose that $c_{v} \neq 0$ (specifically that none of the exponents in Eq. (4.6) are negative). Suppose that there exists a $k$ where $1 \leq k \leq j$ such that $\gamma_{v(j+1-k)} \neq(0,0)$. Without loss of generality, let $k$ be the largest such integer. The fact that $\psi_{S}$ is dense implies that $\left(0, f_{k}-\gamma_{v(j+1-k)}\right)=\left(0, f_{t}\right)$ where $\gamma_{v(j+1-t)}=(0,0)$ for some $t$ (see Eqs. (2.4)-(2.7)). Therefore the exponents of $x_{k}$ and $y_{k}$ are exactly equal to the exponents of $x_{t}$ and $y_{t}$ in Eq. (4.6). Therefore,

$$
\begin{aligned}
& \sum_{\omega \in S_{n}} \operatorname{sign}(\omega) \omega\left(x_{1}^{0-\gamma_{v(1), 1}} y_{1}^{f_{j}-\gamma_{v(1), 2}} x_{2}^{0-\gamma_{v(2), 1}} y_{2}^{f_{j-1}-\gamma_{v(2), 2}}\right. \\
& \left.\quad \cdots x_{j}^{0-\gamma_{v(k), 1}} y_{j}^{f_{1}-\gamma_{v(k), 2}} x_{j+1}^{0-\gamma_{v j(j), 1}} y_{j+1}^{0-\gamma_{v(j+1), 2}} \cdots x_{n}^{g_{n-j-1,1}-\gamma_{v(n), 1}} y_{n}^{0-\gamma_{v(n), 2}}\right)=0 .
\end{aligned}
$$

Similar comments can be made if there exists a $k$ in $j+2 \leq k \leq n$ such that $\gamma_{v(k)} \neq(0,0)$. Therefore, if we have that $\psi_{S}$ is dense and $\gamma \in \Gamma_{\psi_{S}}$ then $m_{\gamma} \in \mathcal{I}_{\mathcal{S}}(X, Y)$. Complete details with all of the calculations can be found in [3].

\section{A $P$-partition type decomposition}

Let $P(X, Y)$ be a monomial such that

$$
P(X, Y)=x_{1}^{p_{1}} y_{1}^{q_{1}} x_{2}^{p_{2}} y_{2}^{q_{2}} \cdots x_{n}^{p_{n}} y_{n}^{q_{n}} \notin \mathcal{I}_{S}(X, Y)
$$

The condition that $P(X, Y) \notin \mathcal{I}_{S}(X, Y)$ implies that $\left(p_{i}, q_{i}\right) \in \mathcal{A}$ for $1 \leq i \leq n$. We define the exponent sequence es $(P,(X, Y))$ of $P(X, Y)$ to be

$$
\operatorname{es}(P(X, Y))=\left(p_{1}, q_{1}\right),\left(p_{2}, q_{2}\right), \ldots,\left(p_{n}, q_{n}\right) .
$$

Label the entries of the sequence $e s(P)$ from smallest to largest with respect to $<_{\mathcal{A}}$ breaking ties by which is farthest left (an example can be found in Eq. (5.14)). With $\phi_{i}$ denoting the 
label of $\left(p_{i}, q_{i}\right)$, set $\phi_{P}$ to be the permutation

$$
\phi_{P}=\left[\begin{array}{ccccc}
1 & 2 & 3 & \cdots & n \\
\phi_{1} & \phi_{2} & \phi_{3} & \cdots & \phi_{n}
\end{array}\right]
$$

and

$$
\sigma_{P}=\left(\phi_{P}\right)^{-1}
$$

Particularly, $\sigma_{P}(i)=k$ implies that $\left(p_{k}, q_{k}\right)$ was labelled $i$.

Let $\left(r_{i, 1}, r_{i, 2}\right)$ denote the entry of $\operatorname{es}(P(X, Y))$ labelled $i$. We can assume that $\left(r_{j, 1}, r_{j, 2}\right)=$ $(0,0)$ or else $P\left(\partial_{X}, \partial_{Y}\right) \Delta_{S}=0$ and $P(X, Y) \in \mathcal{I}_{S}(X, Y)$. With

$$
\psi_{S}=\left[\left[1, \beta_{2}, \ldots, \beta_{j}\right],\left[1, \alpha_{2}, \ldots, \alpha_{n+1-j}\right]\right]
$$

and

$$
\chi_{\sigma_{P}}(i)= \begin{cases}1 & \text { if } \sigma_{P}(i)>\sigma_{P}(i+1) \\ 0 & \text { if } \sigma_{P}(i)<\sigma_{P}(i+1)\end{cases}
$$

for $1 \leq k \leq j-1$, set

$$
\rho_{k}=\left(r_{k, 2}-r_{k+1,2}-\chi_{\sigma_{P}}(k)\right) \quad\left(\bmod \beta_{j+1-k}\right),
$$

for $j+1 \leq h \leq n$, set

$$
\rho_{h}=\left(r_{h, 1}-r_{h-1,1}-\chi_{\sigma_{P}}(h-1)\right) \quad\left(\bmod \alpha_{h+1-j}\right)
$$

and

$$
\rho_{P}=\left[\rho_{1}, \rho_{2}, \ldots, \rho_{j-1}, 0, \rho_{j+1}, \ldots, \rho_{n}\right]
$$

Note that we are considering $\left(\bmod \alpha_{h+1-j}\right)$ and $\left(\bmod \beta_{j+1-k}\right)$ as functions so that $0 \leq \rho_{k} \leq$ $\beta_{j+1-k}-1($ for $1 \leq k \leq j-1)$ and $0 \leq \rho_{h} \leq \alpha_{h+1-j}-1$ (for $\left.j+1 \leq h \leq n\right)$.

With

$$
e s\left(d_{\sigma_{P}, \rho_{P}}^{j}(X, Y)\right)=\left(h_{1,1}, h_{1,2}\right),\left(h_{2,1}, h_{2,2}\right), \ldots,\left(h_{n, 1} h_{n, 2}\right),
$$

let $\left(g_{i, 1}, g_{i, 2}\right)$ denote the entry of $\operatorname{es}\left(d_{\sigma_{P}, \rho_{P}}^{j}\right)$ labelled $i$. Set

$$
\gamma_{i}=\left(\gamma_{i, 1}, \gamma_{i, 2}\right)=\left(r_{i, 1}-g_{i, 1}, r_{i, 2}-g_{i, 2}\right)
$$


and $\gamma_{P}$ to be the sequence

$$
\gamma_{P}=\left[\gamma_{1}, \gamma_{2}, \ldots, \gamma_{n}\right]
$$

with $\gamma_{j}=(0,0)$.

Suppose the entry labelled $j-1$ in $\operatorname{es}(P(X, Y))$ is $\left(0, r_{j-1,2}\right)$. Note that the entry labelled $j$ in $e s(P(X, Y))$ must be $(0,0)$ (or else $\left.P(X, Y) \in \mathcal{I}_{S}(X, Y)\right)$. Now, $\rho_{j-1}=\left(r_{j-1,2}-0-\right.$ $\left.\chi_{\sigma_{P}}(j-1)\right)\left(\bmod \beta_{2}\right)$. Thus

$$
r_{j-1,2}=\rho_{j-1}+f_{i-1} \beta_{2}+\chi_{\sigma_{P}}(j-1)
$$

some nonnegative integer $f_{j-1}$. Now, $g_{j-1,2}$ is the exponent of $y_{\sigma_{P}(j-1)}$ so that

$$
g_{j-1,2}=\rho_{j-1}+\chi_{\sigma_{P}}(j-1)
$$

and

$$
\gamma_{j-1,2}=r_{j-1,2}-g_{j-1,2}=r_{j-1,2}-\rho_{j-1}-\chi_{\sigma P}(j-1)=f_{j-1} \beta_{2}
$$

by Eq. (5.8).

Assume (reverse) inductively that some $k+1$ (where $1 \leq k \leq j-1$ ), we have that

$$
r_{k+1,2}-g_{k+1,2}=\gamma_{k+1,2}=\sum_{i=k+1}^{j-1} f_{i} \beta_{j+1-i}
$$

where each $f_{i}$ is a nonnegative integer. Note that $g_{k, 2}$ is the exponent of $y_{\sigma_{P}(K)}$ and thus Eq. (1.7) implies

$$
g_{k, 2}=g_{k+1,2}+\rho_{k}+\chi_{\sigma_{P}}(k) .
$$

Recall from Eq. (5.4) that

$$
\rho_{k}=\left(r_{k, 2}-r_{k+1,2}-\chi_{\sigma_{P}}(k)\right) \quad\left(\bmod \beta_{j+1-k}\right),
$$

or equivalently,

$$
r_{k, 2}=\rho_{k}+r_{k+1,2}+\chi_{\sigma_{P}}(k)+f_{k} \beta_{j+1-k},
$$

some nonnegative integer $f_{k}$. Using Eq. (5.9), we have

$$
\begin{aligned}
\gamma_{k, 2} & =r_{k, 2}-g_{k, 2} \\
& =\rho_{k}+r_{k+1,2}+\chi_{\sigma_{P}}(k)+f_{k} \beta_{j+1-k}-g_{k+1,2}-\rho_{k}-\chi_{\sigma_{P}}(k)
\end{aligned}
$$




$$
\begin{aligned}
& =r_{k+1,2}+f_{k} \beta_{j+1-k}-g_{k+1,2} \\
& =f_{k} \beta_{j+1-k}+\gamma_{k+1,2} \\
& =\sum_{i=k}^{j-1} f_{i} \beta_{j+1-i}
\end{aligned}
$$

where each $f_{i}$ is a nonnegative integer.

Now $\left(0, \gamma_{k, 2}\right)=e_{j+1-k, 2}$ when $\varepsilon_{i}=f_{j+1-i}$ (recalling Eq. (4.3)) for $2 \leq i \leq j$.

The cases when $k \geq j+1$ are similar. Therefore, we have that

$$
\gamma_{P}=\left[\gamma_{1}, \gamma_{2}, \ldots, \gamma_{n}\right] \in \Gamma_{\psi_{s}}
$$

With $\phi=\phi_{P}$, set

$$
\zeta_{P}=\gamma_{P, \phi}=\left[\gamma_{\phi(1)}, \gamma_{\phi(2)}, \ldots, \gamma_{\phi(n)}\right]
$$

It is easy to see that $\phi_{P}(1)=\phi_{d_{\sigma_{P}, \rho_{P}}}(1), \phi_{P}(2)=\phi_{d_{\sigma_{P}, \rho_{P}}}(2)$, etc. This gives $\phi_{P}=\phi_{d_{\sigma_{P}, \rho_{P}}^{j}}$. Note that if $\sigma(k)=i$ (so that $\phi(i)=\left(\sigma_{P}\right)^{-1}(i)=k$ ) then $\left(p_{i}, q_{i}\right)$ is labelled $k$ in Eq. (5.1) and $\left(h_{i, 1}, h_{i, 2}\right)$ is labelled $k$ in Eq. (5.6). Therefore, we have $\left(p_{i}, q_{i}\right)=\left(r_{k, 1}, r_{k, 2}\right),\left(h_{i, 1}, h_{i, 2}\right)=$ $\left(g_{k, 1}, g_{k, 2}\right)$,

$$
\left(\gamma_{k, 1}, \gamma_{k, 2}\right)=\left(\gamma_{\phi(i), 1}, \gamma_{\phi(i), 2}\right)=\left(\zeta_{i, 1}, \zeta_{i, 2}\right)
$$

and by Eq. (5.7)

$$
x_{i}^{p_{i}} y_{i}^{q_{i}}=x_{\sigma_{k}}^{r_{k, 1}} y_{\sigma_{k}}^{r_{k, 2}}=x_{\sigma_{k}}^{g_{k, 1}} y_{\sigma_{k}}^{g_{k, 2}} x_{\sigma_{k}}^{\gamma_{k, 1}} y_{\sigma_{k}}^{\gamma_{k, 2}}=x_{i}^{h_{i, 1}} y_{i}^{h_{i, 2}} x_{i}^{\zeta_{i, 1}} y_{i}^{\zeta_{i, 2}} .
$$

Thus, we have the following theorem.

Theorem 5.1 Given a sequence

$$
\psi_{S}=\left[\left[1, \beta_{2}, \ldots, \beta_{j}\right],\left[1, \alpha_{2}, \ldots, \alpha_{n+1-j}\right]\right]
$$

and a sequence

$$
P=\left(p_{1,1}, p_{1,2}\right),\left(p_{2,1}, p_{2,2}\right),\left(p_{3,1}, p_{3,2}\right), \ldots,\left(p_{n, 1}, p_{n, 2}\right)
$$

where $\left(p_{i, 1}, p_{i, 2}\right) \in \mathcal{A}$ and $\left(p_{j, 1}, p_{j, 2}\right)=(0,0)$, there is a unique triple $\left(\sigma_{P}, \rho_{P}, \gamma_{P}\right)$, where $\sigma_{P} \in S_{n}, \rho_{P} \in \Xi_{\psi_{S}}$ and $\gamma_{P} \in \Gamma_{\psi_{S}}$, such that if $P=e s(P(X, Y))$ and $\zeta_{P}=\gamma_{P, \phi_{P}}=$ es $(Q(X, Y))$ then

$$
P(X, Y)=d_{\sigma_{P}, \rho_{P}}^{j}(X, Y) Q(X, Y) .
$$

Theorems 4.1 and 5.1 then yield 
Theorem 5.2 If $S$ is dense, if $\left(\sigma_{P}, \rho_{P}, \gamma_{P}\right)$ is the decomposition of $P$ and if $\gamma_{P}$ is not a sequence consisting entirely of entries $(0,0)$ then $m_{\gamma_{P}}(X, Y) \in \mathcal{I}_{S}(X, Y)$.

This decomposition of $e s(P(X, Y))$ in Theorem 5.1 into the triple $\left(e s\left(d_{\sigma_{P}}^{j}\right), \rho_{P}, \gamma_{P}\right)$ is a generalization of a $P$-partition decomposition given in [10] (see page 213). It will reduce to exactly this $P$-partition decomposition when $\psi_{S}=[[1,1, \ldots, 1]$, Ø] (forcing $\left.\rho_{P}=[0,0, \ldots, 0]\right)$. Note that in this case, the requirement that $\left(r_{j, 1}, r_{j, 2}\right)=(0,0)$ (the entry in Eq. (5.1) labelled $j$ ) is not needed (see Section 7).

Example With $n=8, \psi_{S}=[[1,1,3,3],[1,1,2,2,4]]$ (which implies $j=4$ ) and

$$
P(X, Y)=x_{1}^{5} x_{2}^{23} y_{3}^{9} y_{4}^{21} y_{5}^{1} x_{6}^{15} x_{8}^{1}
$$

then $e s(P(X, Y))$ with its labelling is

$$
\operatorname{es}(P(X, Y))=(5,0)_{6},(23,0)_{8},(0,9)_{2},(0,21)_{1},(0,1)_{3},(15,0)_{7},(0,0)_{4},(1,0)_{5} \text {. }
$$

Now,

$$
\begin{aligned}
\phi_{P} & =\left[\begin{array}{llllllll}
1 & 2 & 3 & 4 & 5 & 6 & 7 & 8 \\
6 & 8 & 2 & 1 & 3 & 7 & 4 & 5
\end{array}\right], \\
\sigma_{P} & =\left[\begin{array}{llllllll}
1 & 2 & 3 & 4 & 5 & 6 & 7 & 8 \\
4 & 3 & 5 & 7 & 8 & 1 & 6 & 2
\end{array}\right] .
\end{aligned}
$$

and

$$
\begin{aligned}
\rho= & {[21-9-1(\bmod 3), 9-1-0(\bmod 3), 1-0-0(\bmod 1), 0,} \\
& 1-0-0(\bmod 1), 5-1-1(\bmod 2), 15-5-0(\bmod 2), \\
& 23-15-1(\bmod 4)] \\
= & {[2,2,0,0,0,1,0,3] . }
\end{aligned}
$$

Note

$$
d_{\sigma, \rho}^{4}(X, Y)=\left(y_{4}^{2}\right)\left(y_{3}^{2} y_{4}^{2}\right)\left(y_{4}\right)\left(x_{1} x_{6} x_{2}\right)\left(x_{2}^{3}\right)\left(x_{1} x_{6} x_{2}\right)\left(x_{2}\right)=x_{1}^{2} x_{2}^{6} y_{3}^{2} y_{4}^{5} x_{6}^{2},
$$

$e s\left(d_{\sigma, \rho}^{4}(X, Y)\right)$ (along with its exponent labelling) is

$$
e s\left(d_{\sigma, \rho}^{4}(X, Y)\right)=(2,0)_{6},(6,0)_{8},(0,2)_{2},(0,5)_{1},(0,0)_{3},(2,0)_{7},(0,0)_{4},(0,0)_{5}
$$

and

$$
\gamma_{P}=[(0,16),(0,7),(0,1),(0,0),(1,0),(3,0),(13,0),(17,0)] \in \Gamma_{\psi_{s}}
$$


Note that this corresponds to $\varepsilon_{2}=1, \varepsilon_{3}=2, \varepsilon_{4}=3, \theta_{2}=1, \theta_{3}=1, \theta_{4}=5$ and $\theta_{5}=1$ in Eqs. (4.3) and (4.4).

$$
\zeta_{P}=\gamma_{P, \phi}=[(3,0),(17,0),(0,7),(0,16),(0,1),(13,0),(0,0),(1,0)] .
$$

\section{Spanning}

The type of $P(X, Y)$, denoted by $\tau(P(X, Y))$, is the rearrangement of the exponent sequence es $(P(X, Y))$ in increasing with respect to $<_{\mathcal{A}}$. For any two sequences $\mu=\left[\mu_{1}, \mu_{2}, \ldots, \mu_{n}\right]$ and $v=\left[v_{1}, v_{2}, \ldots, v_{n}\right]$ of length $n$ with entries from the set $\mathcal{A}$, we will say that $\mu<_{\mathcal{A}} v$ whenever $\mu_{i}=v_{i}$ for $1 \leq i \leq k-1$ and $\mu_{k}<\mathcal{A} v_{k}$. We will say that $P(X, Y)<_{t e} Q(X, Y)$ whenever

1. $\tau(P(X, Y))<_{\mathcal{A}} \tau(Q(X, Y))$;

2. if $\tau(P(X, Y))=\tau(Q(X, Y))$ then $\operatorname{es}(P(X, Y))<_{\mathcal{A}} \operatorname{es}(Q(X, Y))$.

The largest monomial $N(X, Y)$ with respect to $<_{t e}$ such that $N(X, Y) \notin \mathcal{I}_{S}(X, Y)$ is the monomial

$$
N(X, Y)=x_{1}^{a_{n}} x_{2}^{a_{n-1}} \cdots x_{n-j}^{a_{j+1}}
$$

where $S$ is listed with respect to increasing order of $<_{\mathcal{A}}$ as in Eq. (1.5). Note that $N(X, Y)=$ $d_{\sigma, \rho}^{j}(X, Y)$ where

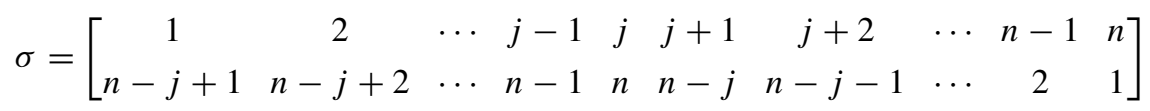

and $\rho=\left[0,0, \ldots, 0, \alpha_{2}-1, \alpha_{3}-1 \cdots, \alpha_{n+j-1}-1\right]$. Particularly, $N(X, Y) \in \mathcal{D}_{S}$.

Let $P(X, Y) \notin \mathcal{I}_{S}(X, Y)$ be the monomial that is largest with respect to $<_{t e}$ such that $P(X, Y)$ is not in the span of $\mathcal{D}_{S}$. Since $P(X, Y) \notin \mathcal{D}_{S}$ then $\gamma_{P} \in \Gamma_{\psi_{S}}$ and (from Eq. (5.11) and Theorem 5.2)

$$
m_{\zeta P}(X, Y)=m_{\gamma_{P, \phi}}(X, Y)=m_{\gamma_{P}}(X, Y) \in \mathcal{I}_{S}(X, Y)
$$

Now, using the notation of Eqs. (5.6) and (5.11) and setting $\sigma=\sigma_{P}$ and $\rho=\rho_{p}$, we have

$$
\begin{aligned}
& m_{\zeta_{P}}(X, Y) d_{\sigma, \rho}^{j}(X, Y) \\
& =\left(\sum_{\nu \in S_{n}} x_{1}^{\zeta_{\nu_{1}, 1}} y_{1}^{\zeta_{1}, 2} x_{2}^{\zeta_{\nu_{2}, 1}} y_{2}^{\zeta_{\nu_{2}, 2}} \cdots x_{n}^{\zeta_{\nu_{n}, 1}} y_{n}^{\zeta_{\nu_{n}, 2}}\right) x_{1}^{h_{1,1}} y_{1}^{h_{1,2}} x_{2}^{h_{2,1}} y_{2}^{h_{2,2}} \cdots x_{n}^{h_{n, 1}} y_{n}^{h_{n, 2}}
\end{aligned}
$$

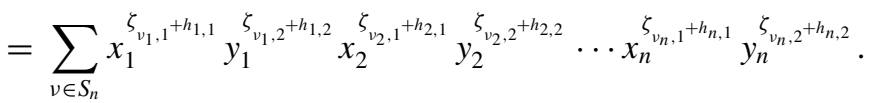


Note that we have the following cases.

1. If for some $i$ (with $1 \leq i \leq n)\left(\zeta_{v_{i}, 1}+h_{i, 1}, \zeta_{v_{i}, 2}+h_{i, 2}\right) \notin \mathcal{A}$ then

$$
x_{1}^{\zeta_{\nu_{1}, 1}+h_{1,1}} y_{1}^{\zeta_{1}, 2+h_{1,2}} x_{2}^{\zeta_{\nu_{2}, 1}+h_{2,1}} y_{2}^{\zeta_{\nu_{2}, 2}+h_{2,2}} \cdots x_{n}^{\zeta_{\nu_{n}, 1}+h_{n, 1}} y_{n}^{\zeta_{\nu_{n}, 2}+h_{n, 2}} \in \mathcal{I}_{S}(X, Y)
$$

2. $x_{1}^{\zeta_{1,1}+h_{1,1}} y_{1}^{\zeta_{1,2}+h_{1,2}} x_{2}^{\zeta_{2,1}+h_{2,1}} y_{2}^{\zeta_{2,2}+h_{2,2}} \cdots x_{n}^{\zeta_{n, 1}+h_{n, 1}} y_{n}^{\zeta_{n, 2}+h_{n, 2}}=P(X, Y)$.

3. If $\left(h_{i, 1}, h_{i, 2}\right)<_{\mathcal{A}}\left(h_{k, 1}, h_{k, 2}\right)$ then $\left(\zeta_{i, 1}, \zeta_{i, 2}\right) \leq_{\mathcal{A}}\left(\zeta_{k, 1}, \zeta_{k, 2}\right)$.

4. If $\left(h_{i, 1}, h_{i, 2}\right)=\left(h_{k, 1}, h_{k, 2}\right)$ and $i<k$ then $\phi_{i}<\phi_{k}$ (from Eq. (5.2)) and $\left(\zeta_{i, 1}, \zeta_{i, 2}\right) \leq{ }_{\mathcal{A}}$ $\left(\zeta_{k, 1}, \zeta_{k, 2}\right)$

5. If $\left(h_{i, 1}, h_{i, 2}\right)<_{\mathcal{A}}\left(h_{k, 1}, h_{k, 2}\right),\left(m_{i, 1}, m_{i, 2}\right)<_{\mathcal{A}}\left(m_{k, 1}, m_{k, 2}\right)$ and if both $\left(h_{i, 1}+m_{i, 1}, h_{i, 2}+\right.$ $\left.m_{i, 2}\right) \in \mathcal{A}$ and $\left(h_{k, 1}+m_{k, 1}, h_{k, 2}+m_{k, 2}\right) \in \mathcal{A}$ then $\left(h_{i, 1}+m_{i, 1}, h_{i, 2}+m_{i, 2}\right)<_{\mathcal{A}}\left(h_{k, 1}+\right.$ $\left.m_{k, 1}, h_{k, 2}+m_{k, 2}\right)$.

Let

$$
F=\left\{\left(f_{1,1}, f_{1,2}\right),\left(f_{2,1}, f_{2,2}\right), \ldots,\left(f_{v, 1}, f_{v, 2}\right)\right\}
$$

denote the collection of elements of $\mathcal{A}$ that make up the entries of $e s\left(d_{\sigma, \rho}^{j}(X, Y)\right)$ listed in increasing order with respect to $<_{\mathcal{A}}$. Let

$$
K_{i}=\left\{k_{1}, k_{2}, \ldots, k_{q_{i}}\right\}
$$

be the collection of integers such that $\left(h_{k_{m}, 1}, h_{k_{m}, 2}\right)=\left(f_{i, 1}, f_{i, 2}\right)$. Set $\eta_{i}$ to be the ordered sequence

$$
\eta_{i}=\left[\left(\zeta_{k_{1}, 1}, \zeta_{k_{1}, 2}\right),\left(\zeta_{k_{2}, 1}, \zeta_{k_{2}, 2}\right), \ldots,\left(\zeta_{q_{i}, 1}, \zeta_{q_{i}, 2}\right)\right]
$$

of all the elements in $\zeta_{P}$ (see Eq. (5.11)) such that

$$
\left(h_{k_{m}, 1}, h_{k_{m}, 2}\right)=\left(f_{i, 1}, f_{i, 2}\right)
$$

listed so that $k_{i}<k_{i+1}$ (recall that $\left(h_{i, 1}, h_{i, 2}\right)$ are defined in Eq. (5.6)). By Property 4, we have that

$$
\left(\zeta_{k_{i}, 1}, \zeta_{k_{i}, 2}\right) \leq_{\mathcal{A}}\left(\zeta_{k_{i+1}, 1}, \zeta_{k_{i+1}, 2}\right)
$$

for $1 \leq i \leq q_{i}-1$. Set

$$
\eta_{i, v}=\left[\left(\zeta_{v_{k_{1}}, 1}, \zeta_{v_{k_{1}}, 2}\right),\left(\zeta_{v_{k_{2}}, 1}, \zeta_{v_{k_{2}}, 2}\right), \ldots,\left(\zeta_{v_{k_{i}}, 1}, \zeta_{v_{k_{i}}, 2}\right)\right]
$$

where $v \in S_{n}$. 
Example With $d_{\sigma, \rho}^{4}$ given in Eq. (5.15), we have

$$
F=\{(0,5),(0,2),(0,0),(2,0),(6,0)\}
$$

Thus $\left(f_{1,1}, f_{1,2}\right)=(0,5)$ and $K_{1}=\{4\}$. Similarly, $\left(f_{3,1}, f_{3,2}\right)=(0,0)$ and $K_{3}=\{5,7,8\}$. Therefore $\eta_{1}=[(0,16)]$ and $\eta_{3}=[(0,1),(0,0),(1,0)]$. With $v$ a permutation such that

$$
v=\left[\begin{array}{llllllll}
1 & 2 & 3 & 4 & 5 & 6 & 7 & 8 \\
3 & 6 & 8 & 2 & 5 & 1 & 4 & 7
\end{array}\right]
$$

we have

$$
\eta_{1, v}=\left[\zeta_{P, v(4)}\right]=[(17,0)]
$$

Similarly,

$$
\eta_{3, v}=[(0,1),(0,16),(0,0)] .
$$

On the other hand, if

$$
\mu=\left[\begin{array}{llllllll}
1 & 2 & 3 & 4 & 5 & 6 & 7 & 8 \\
1 & 2 & 3 & 4 & 7 & 6 & 8 & 5
\end{array}\right]
$$

then

$$
\eta_{3, \mu}=[(0,0),(1,0),(0,1)]
$$

Note that $\eta_{3, \mu}$ is a permutation of the sequence $\eta_{3}$ while the sequence $\eta_{3, v}$ is not a permutation of the sequence $\eta_{3}$ (i.e., $\eta_{3, v}$ has different entries than $\eta_{3}$ ).

If $\eta_{i}=\eta_{i, v}$ (as an ordered sequence) for all $1 \leq i \leq v$ then we have (by Property 2) that

$$
\begin{aligned}
& x_{1}^{\zeta_{\nu_{1}, 1}+h_{1,1}} y_{1}^{\zeta_{\nu_{1}, 2}+h_{1,2}} x_{2}^{\zeta_{\nu_{2}, 1}+h_{2,1}} y_{2}^{\zeta_{\nu_{2}, 2}+h_{2,2}} \cdots x_{n}^{\zeta_{\nu_{n}, 1}+h_{n, 1}} y_{n}^{\zeta_{\nu_{n}, 2}+h_{n, 2}} \\
& =x_{1}^{\zeta_{1,1}+h_{1,1}} y_{1}^{\zeta_{1,2}+h_{1,2}} x_{2}^{\zeta_{2,1}+h_{2,1}} y_{2}^{\zeta_{2,2}+h_{2,2}} \cdots x_{n}^{\zeta_{n, 1}+h_{n, 1}} y_{n}^{\zeta_{n, 2}+h_{n, 2}} \\
& =P(X, Y) .
\end{aligned}
$$

If each $\eta_{i, v}$ is a non-identity permutation of the respective sequence $\eta_{i}$ for all $1 \leq i \leq v$ then

$$
\begin{aligned}
& \tau\left(x_{1}^{\zeta_{\nu_{1}, 1}+h_{1,1}} y_{1}^{\zeta_{\nu_{1}, 2}+h_{1,2}} x_{2}^{\zeta_{\nu_{2}, 1}+h_{2,1}} y_{2}^{\zeta_{\nu_{2}, 2}+h_{2,2}} \cdots x_{n}^{\zeta_{v_{n}, 1}+h_{n, 1}} y_{n}^{\zeta_{\nu_{n}, 2}+h_{n, 2}}\right) \\
& \quad=\tau(P(X, Y)) .
\end{aligned}
$$


but by Property 4, we have that

$$
\begin{aligned}
& \operatorname{es}\left(x_{1}^{\zeta_{\nu_{1}, 1}+h_{1,1}} y_{1}^{\zeta_{\nu_{1}, 2}+h_{1,2}} x_{2}^{\zeta_{\nu_{2}, 1}+h_{2,1}} y_{2}^{\zeta_{\nu_{2}, 2}+h_{2,2}} \cdots x_{n}^{\zeta_{\nu_{n}, 1}+h_{n, 1}} y_{n}^{\zeta_{\nu_{n}, 2}+h_{n, 2}}\right) \\
& \quad>_{\mathcal{A}} \operatorname{es}(P(X, Y)) .
\end{aligned}
$$

Finally, suppose $\eta_{i, v}$ is not a permutation of the sequence $\eta_{i}$ for some $i$. By considering the smallest integer $m$ such that $\eta_{m, v}$ is not a permutation of the sequence $\eta_{m}$, it is not difficult to see that Property 3 implies

$$
\begin{aligned}
& \tau\left(x_{1}^{\zeta_{\nu_{1}, 1}+h_{1,1}} y_{1}^{\zeta_{\nu_{1}, 2}+h_{1,2}} x_{2}^{\zeta_{\nu_{2}, 1}+h_{2,1}} y_{2}^{\zeta_{\nu_{2}, 2}+h_{2,2}} \cdots x_{n}^{\zeta_{v_{n}, 1}+h_{n, 1}} y_{n}^{\zeta_{\nu_{n}, 2}+h_{n, 2}}\right) \\
& \quad>_{\mathcal{A}} \tau(P(X, Y)) .
\end{aligned}
$$

Thus we have

Theorem 6.1 Let $\psi_{S}$ be dense, let $P(X, Y)$ be a monomial such that $P(X, Y) \notin \mathcal{I}_{S}(X, Y)$ and let $\left(\sigma_{P}, \rho_{P}, \gamma_{P}\right)$ be the generalized $P$-partition decomposition of

$$
P=e s(P(X, Y))
$$

given by Theorem 5.1. Then

$$
\begin{aligned}
m_{\gamma_{P, \sigma}}(X, Y) d_{\sigma_{P, \rho}}^{j}(X, Y) \equiv & c_{P} P(X, Y) \\
& +\sum_{P(X, Y)<_{t e} Q(X, Y)} c_{Q} Q(X, Y) \quad\left(\bmod \mathcal{I}_{S}(X, Y)\right)
\end{aligned}
$$

where $c_{P} \neq 0$. Therefore, in $\mathbb{C}_{S}[X, Y]$ we have

$$
P(X, Y) \equiv-\frac{1}{c_{P}} \sum_{P(X, Y)<t_{e} Q(X, Y)} c_{Q} Q(X, Y) \quad\left(\bmod \mathcal{I}_{S}(X, Y)\right)
$$

Since we assumed that $P(X, Y)$ is the largest monomial with respect to $<_{t e}$ such that $P(X, Y) \notin \mathcal{I}_{S}(X, Y)$ and $P(X, Y)$ is not in the span of $\mathcal{D}_{S}$, Theorem 6.1 yields a contradiction. Thus $P(X, Y)$ must be in the span of $\mathcal{D}_{S}$ and combining this with Theorem 3.2 yields Theorem 1.1. Specifically,

Theorem 6.2 Let $S$ be a dense sequence. The collection

$$
\mathcal{D}_{S}=\left\{d_{\sigma, \rho}^{j}(X, Y): \sigma \in S_{n}, \rho \in \Xi_{\psi_{S}}\right\}
$$

is a basis for $\mathbb{C}_{S}[X, Y]$ with coefficients from $\mathbb{C}$. 


\section{Notes about the one-variable case}

When

$$
\psi_{S}=\left[\emptyset,\left[\beta_{1}, \beta_{2}, \ldots, \beta_{n}\right]\right]
$$

or

$$
\psi_{S}=\left[\left[\alpha_{1}, \alpha_{2}, \ldots, \alpha_{n}\right], \emptyset\right],
$$

$\triangle_{S}(X, Y)$ reduces to a determinant in a single collection of variables $Y=\left\{y_{1}, \ldots, y_{n}\right\}$ or $X=\left\{x_{1}, \ldots, x_{n}\right\}$, respectively. Note that in these cases, we do not require $\beta_{1}=1$ or $\alpha_{1}=1$. The definition of dense in these two cases in exactly the same as that described in Eqs. (2.2)-(2.7). We define the collection $\Xi_{\psi_{s}}$ as

$$
\Xi_{\psi_{S}}=\left\{\left[\rho_{1}, \ldots, \rho_{n}\right]\right\}_{\rho},
$$

where either $0 \leq \rho_{k}<\beta_{n+1-k}$, for $1 \leq k \leq n$ (in case (7.1)) or $0 \leq \rho_{h}<\alpha_{h}$, for $1 \leq h \leq n$ (in case (7.2)).

The analogous descent monomials are defined as

$$
d_{\sigma, \rho}^{j}(X, Y)=\left(\prod_{k=1}^{n} y_{\sigma(1)}^{\rho_{k}} y_{\sigma(2)}^{\rho_{k}} \cdots y_{\sigma(k)}^{\rho(k)}\right)\left(\prod_{\substack{1 \leq i \leq n-1 \\ \sigma(i)>\sigma(i+1)}} y_{\sigma(1)} y_{\sigma(2)} \cdots y_{\sigma(i)}\right)
$$

and

$$
d_{\sigma, \rho}^{j}(X, Y)=\left(\prod_{k=1}^{n} x_{\sigma(k)}^{\rho_{k}} x_{\sigma(k+1)}^{\rho_{k}} \cdots x_{\sigma(n)}^{\rho_{k}}\right)\left(\prod_{\substack{1 \leq i \leq n-1 \\ \sigma(i)>\sigma(i+1)}} x_{\sigma(i+1)} \cdots x_{\sigma(n)}\right),
$$

respectively. Note that in fact these monomials correspond directly with $P$-partitions (see [10]).

The definition for a cocharge tableaux is the same as that given in Section 3, except step A is replaced by

$\mathrm{A}^{\prime}$. With $\psi_{S}$ given by (7.2) (or $\psi_{S}$ given by Eq. (7.1), respectively) and $\rho=\left(\rho_{1}, \rho_{2} \ldots, \rho_{n}\right)$ then replace 1 (or $n$ respectively) by $c_{1}=\left(c_{1,1}, c_{1,2}\right)=\left(\rho_{1}, 0\right)\left(\right.$ or $c_{n}=\left(c_{n, 1}, c_{n, 2}\right)=$ $\left(0, \rho_{n}\right)$, respectively).

The analogues for $\Gamma_{\psi_{s}}$ are that we set

$$
\Gamma_{\psi_{S}}=\left\{\left(e_{n}, e_{n-1}, \ldots, e_{2}, e_{1}\right): \varepsilon_{1}+\cdots+\varepsilon_{n}>0\right\}
$$

in situation (7.1), and

$$
\Gamma_{\psi_{s}}=\left\{\left(h_{1}, h_{2}, \ldots, h_{n}\right): \theta_{1}+\cdots+\theta_{n}>0\right\}
$$


in situation (7.2), where $\varepsilon_{k}$ and $\theta_{h}$ are nonnegative integers,

$$
e_{k}=\left(0, \sum_{i=1}^{k} \varepsilon_{i} \beta_{i}\right)
$$

(for $1 \leq k \leq n$ ) and

$$
h_{g}=\left(\sum_{i=1}^{g} \theta_{i}, \alpha_{f}, 0\right)
$$

(for $1 \leq g \leq n$ ) respectively.

The analogues for Theorems 3.2, 5.1, 5.2, 6.1 and 6.2 follow immediately.

\section{References}

1. E.E. Allen, "The descent monomials and a basis for the diagonally symmetric polynomials," J. Alg. Combin. 3 (1994), 5-16.

2. E.E. Allen, "Some graded representations of the complex reflection groups," J. Combin. Theory, Ser. A $\mathbf{8 7}$ (1999), 287-332.

3. E.E. Allen, "Bitableaux bases for some Garsia-Haiman modules and other related modules," Elect. J. Combin. 9 (2002), R36, 1-59.

4. A.M. Garsia, "Combinatorial methods in the theory of Cohen-Macaulay rings," Adv. Math. 38 (1980), 229266.

5. A.M. Garsia and M.D. Haiman, "A graded representation model for Macdonald's polynomials," Proc. Natl. Acad. Sci. USA 90 (April 1993), 3607-3610.

6. D.E. Knuth, The Art of Computer Programming, Vol. 1: Fundamental Algorithms, Addison-Wesley, Reading, MA, 1968.

7. H. Morita and H.-F. Yamada, "Higher Specht polynomials for the complex reflection group $G(r, p, n)$," Hokkaido Mathematical Journal 27 (1998), 505-515.

8. V. Riener, "Quotients of Coxeter Complexes and P-partitions," Mem. Amer. Math Soc. 95 (460), 1992.

9. B.E. Sagan, The Symmetric Group, Wadsworth \& Brooks/Cole, Monterey, 1991.

10. R.P. Stanley, Enumerative Combinatorics, Vol. 1, Wadsworth \& Brooks/Cole, Monterey, 1986.

11. J.R. Stembridge, "On the eigenvalues of representations of reflection groups and wreath products," Pacific J. Math. 140 (1989), 353-396. 